\title{
Efectos de un programa de entrenamiento aeróbico de dos años de duración sobre el perfil lipídico de escolares obesos
}

\author{
Victor Casanueva E. ${ }^{1}$; Cristian Milos $\mathrm{G}^{2}{ }^{2}$; María Teresa Chiang $\mathrm{S} .^{3}$; \\ Valentina Espejo G. ${ }^{1}$; Ximena Cid $\mathrm{C}^{4}{ }^{4}$; Grimanesa Riquelme $\mathrm{M}^{5}$ \\ Effects of two years aerobic training on the lipid \\ profile in obese school children
}

\begin{abstract}
The incidence of cardiovascular risk factors and the effect of a two years aerobic training programme in 30 obese school children of both sexes (aged 6 to 12 ) were evaluated. Training consisted in thrae veekly 60 min sessions of aerobic exercise aimed to atain an estimated 60 to $70 \%$ of calculated maximal heart rate, three times per week. Information about eating habits and diet was also supplied to patients and their parents. At the end of two years only seven children were still in the program for evaluation. Their results were increased estimatex physical work canacity at heart rate 170 beats per min (PWC 170) from 495 to $615 \mathrm{~km} / \mathrm{min}$; reducticn of percent body fat (from 30.5 to $27.4 \%$ ), of total serum cholesterol ifrom 181 to $148 \mathrm{mg} / \mathrm{di}$ and of LDL-C ifrom 106 to $84 \mathrm{mg} / \mathrm{dli}$. All these changes were significant $t \mathrm{p}<0.05 t$. As a conclusion, this programme improved fitness, reduced body fat as well as cardiovascular risk factors.
\end{abstract}

(Key words: Lipoproteins, HDL cholesteral, LDL, excercise, obesity.)

Las complicaciones cardiacas de la ateroesclerosis comienzan a manifestarse generalmente alrededor de la cuarta década de la vida. Sin embargo, la prevención de las enfermedades cardiovasculares debe comenzar en la niñez, debido a que en esta época de la vida se inicia la ateroesclerosis ${ }^{1}$ y al mismo tiempo se adquieren hábitos que influirán en el estilo de vida futuro.

1. Departamento de Pediatría, Facultad de Medicina, Universidad de Concepción.

2. Químico Farmacéutico. Departamento de Bioquímia Clínjea e Inmunología, Facultad de Farmacia, Universidad de Concepción.

3. Bioquímico. Laboratorio de Etgonomia, Departamento de Fisiopatología, Facultad de Ciencias Biológicas y Recursos Naturales, Universidad de Concepción.

4. Bioquímico. Laboratorio Central, Hospital Guillermo Grant Benavente, Concepción.

5. Profesora. Laboratorio de Ergonomía, Departamento de Fisiopatología, Facultad de Ciencias Biológicas y Recursos Naturales, Universidad de Concepción.

Trabajo financiado por la Direción de Investigación de la Universidad de Concepción, Proy ecto 21.08.10.
El comitè de expertos de la OMS, en prevención de la cardiopatía coronaria, recomienda una estrategia de población destinada a modificar el estilo de vida, siendo las in tervenciones a nivel escolar las de mayor rendimiento ${ }^{2}$. La obesidad constituye un factor de riesgo cardiovascular, considerado como independiente, según el estudio de Framingham ${ }^{3}$, el que favorece el desarrollo, en forma asociada o no, de los factores llanados primarios, tales como la hipercolesterolemia e hipertensión asterial. En escolares de Concepción se han estudiado dichos factores de riesgo, habiéndose publicado los resultados de mediciones de los lípidos séricos 4 .

Aplicando la estrategia recomendada por la OMS, se solicitó la participación voluntaria de un grupo de niños obesos para determinar en ellos la incidencia de factores de riesgo cardiovascular y las variaciones observadas luego de un programa de dos años de duración, basado en recomendaciones dietéticas tendientes a modificar los hábitos alimentarios; ejercicios aeróbicos vigilados y reuniones periódicos con los niños y sus padres para estimular los cambios de conducta y analizar el desarrollo del programa. 


\section{Material y Método}

Se tomó una muestra al azar de 30 escolares cuya edad promedio fue 9,3 años (rango 6 a 12 años), de anbos sexos, pertenecientes al grupo clasificado como de alto riesgo en una publicación anterior (obesos e hipercolesterolémicos) ${ }^{4}$. Durante el desarrollo del programa, hubo un gran porcentaje de desercion, terminando los dos años sólo 7 de los niños iniciales: cinco hombres $y$ dos mujeres.

Previa autorización de los padres, y luego de charlas informativas, los niños seleccionados fueron examinados por pediatras, con examen físico completo y evaluación del estado nutricional. Para las medicjones de peso, talla y pliegues de grasa subcutánea se utilizaron los procedimientos ya descritos 5 . La presión arterial se mjaió según normas comunes de referencia ${ }^{6}$ y la determina. ción de los lipidos séricos se realizó según el método descrito anterjormente ${ }^{4}$. El contenido de masa grasa ( $\%$ MG) se calculó en base al gosor de cuatro pbiegues subcutáneos: bicipital, tricipital, subescapular y suprailáaco, medidos con calibrador marca Holtain, según ecuaciones de Brook ${ }^{7}$ y $\mathrm{Siri}^{8}$.

Se catalogó como obeso a los niños cuyo peso era mayor que dos desviaciones estándar del peso ideal para su edad y talla, según curvas y tablas chilenas" y cuyo contenido de masa grasa exced ía de $20 \%$ del peso corporal, en nifios, y $30 \%$ en nifias ${ }^{10}$.

La capacidad física aeróbica de los escolares se calculó como la capacidad de trabajo físico 170 (CTF170), a partir de las frecuencias cardíacas registradas para tres cargas submáximas de intensidad creciente, de cinco minutos de duración, aplicadas en un cicloergómetro marca Body Guard 990 . El consumo máximo de oxígeno se estimó mediante el nomograma de Astrand ${ }^{11}$, utilizando la frecuencia cardiaca alcanzada para la mayor de las tres cargas aplicadas.

Siguiendo las recomendaciones del programa cl ínico de investigación para lípidos del Instituto Nacional de Salud de EUA, se consideraron factores de riesgo: colesterol sérico igual o mayor a $175 \mathrm{mg} / \mathrm{dl}$; colesterol ligado a lipoproteínas de baja densidad (C-LDL) igual o mayor a $130 \mathrm{mg} / \mathrm{dl}$; colesterol ligado a lipoprotejnas de alta densidad (C-HDL) menor de $35 \mathrm{mg} / \mathrm{dl}^{12}$; presión arterial sistólica (PAS) mayor a $120 \mathrm{mmHg}$; presión arterial diastólica (PAD) mayor a $80 \mathrm{mmHg}$ e historia familiar de cardiopatía coronaria en familiares consanguíneos menores de $\$ 5$ años.

E1 entrenamiento aeróbico fue diseñado y aplicado por un profesor de educación física entre los años 1990 y 1991 . Se realizó tres veces a la semana, en sesiones de 60 minutos de trabajo aeróbico con intensidad promedio entre 60 y $70 \%$ de la frecuencia caróíaca máxima calculada. En consideración a la edad de los participantes, el entrenamiento se hizo en base a juegos, caminatas al aire libre, trabajo en pendientes y ompetencias. Los períodos de vacaciones fueron respetados, realizando una evaluación al término de ellas, con el fin de constatar posibles modificaciones de los parámetros evaluados. Cada 15 sesiones se realizaron controles de la capacidad física, con el fin ue ajustar la intensidad del programa de acondicionamiento físico. Se realizó, además, tratamiento conductual de la obesidad ${ }^{13}$. Durante los seis primeros meses, cada niño llevó un cuaderno para anotar su ìnesta diaria de alimentos, el que fue tevisado semanalmente; transcurrida la primera etapa, el control se rcalizó una vez al mes. Las entrevistas con el equipo de salud reunían a padres y a niños, comentando en conjuntu los progresos obtenidos, $y$ entregando est ímulos a los de mejor desempeño. La instrucción nutricional, más que obligar a tuna dieta fija, se cuñó a las recomendaciones de la Academia Americana de Pediatría, disminuyendo el consumo de grasas saturadas e instruyendo a padres y niñox sobre el contenido de grasa animal de los alimentos. Se mantuvieron aportes de $30 \%$ del total de las calor ias en base a grasas, necesarias para el crecimiento ${ }^{14}$.

El análisis de los resultados se realizó con un programa estadístico SYSTAT versión 3 (The System for Statistics) en un computador Acer 915. Para el análisis de significancia se aplicó la prueba t de Student para datos pareados.

\section{Resultados}

El seguiniento fue completado por sólo 7 de 30 niños incorporados inicialmente. En la tabla 1 se describen los valores (promedio y desviación estándar) antropométricos de los siete escolares participantes que terminaron el estudio ( $\bar{x}$ y DE), antes y después de los dos años de transcurrido el programa. Como se puede observar, hubo un aumento significativo del peso y la estatura ( $\mathrm{p}<0,05$ ), lo que corresponde a variaciones propias del crecimiento. En cuanto a la suma de los pliegues de grasa subcutánea y al porcentaje de masa grasa, ambos parámetros disminuyeron significativamente $(\mathrm{p}<0,05)$ durante el mismo periodo. Por su parte, la tabla 2 muestra el promedio y la desviación estándar de la capacidad física y el perfil lipídico inicial y final, valores que se modificaron significativamente, a excepción de los triglicéridos y la relación colesterol total/colesterol HDL: CT/C.HDL. La presión arterial no mostró cambios signifícativos, haciendo notar que todos los niños incluidos en el estudio tenian presión arterial en rangos normales a) ingresar.

\section{Comentario}

Por definición los niños incorporados al estudio eran obesos ${ }^{9}, 10 \mathrm{y}$, además, su perfil lipídj. co estaba alterado. La capacidad de trabajo físico de estos niños (PWC-170) y VO2 máx estimada, fue inferior a los de niños de edades similares no sedentarios o deportistas ${ }^{5}$. Al res. pecto, es importante señalar que al evaluar niños obesos, que por lo general llevan una vida 
Tabla 1

Características antropométricas de sicte escolares obesos al inicio y término de un programa de dos años de entrenamiento aeróbico y dieta

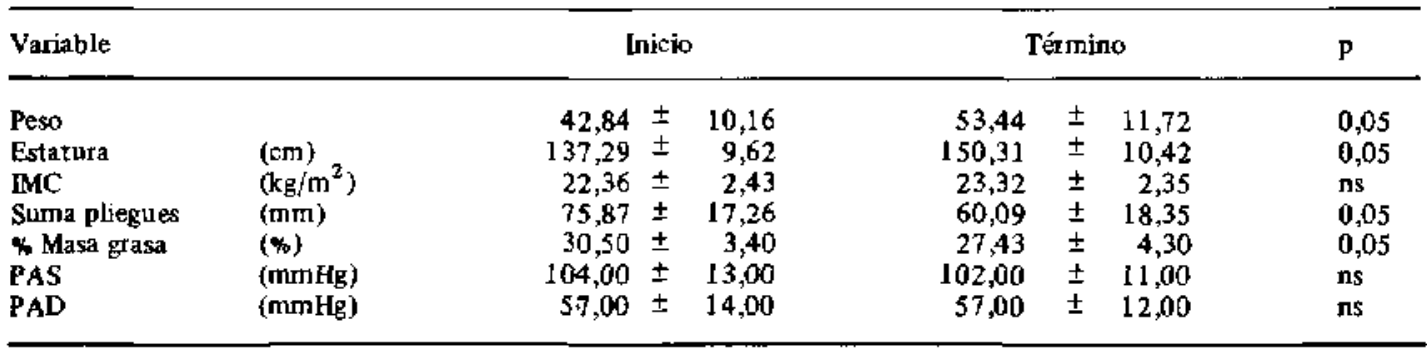

Los valores son promedio \pm desviación estándar.

Tabla 2

Capacidad de trabajo y perfil lipídico de siete escolares obesos, al inicio y después de dos años de entrenamiento aeróbico y dieta

\begin{tabular}{|c|c|c|c|c|c|c|}
\hline \multicolumn{2}{|l|}{ Variable } & \multicolumn{2}{|c|}{ Inicio } & \multicolumn{2}{|c|}{ Término } & \multirow{2}{*}{$\begin{array}{c}\mathbf{p} \\
<0,05\end{array}$} \\
\hline PWC-170 & $(\mathrm{kgm} / \mathrm{min})$ & $490,60=$ & 107,10 & 614,80 & $\pm 129,90$ & \\
\hline VO2 max & & $1,30=$ & 0,26 & 1,87 & 0,38 & $<0,05$ \\
\hline Colesterol & $(\mathrm{mg} / \mathrm{dl})$ & 181,00 & 14,30 & 148,40 & $\pm \quad 16,50$ & $<0,05$ \\
\hline C-HDL & (mg/dl) & $54,30=$ & 16,50 & 45,60 & $\pm \quad 11,10$ & $<0,05$ \\
\hline C-LDL & $(\mathrm{mg} / \mathrm{dl})$ & $106,30=$ & 15,60 & 83,60 & $\pm \quad 20,80$ & $<0,05$ \\
\hline Triglicéridos & $\{\mathrm{mg} / \mathrm{dl}\}$ & $97,60=$ & 20,00 & 97,60 & $\pm \quad 27,30$ & ns \\
\hline CT/C-HDL & & $3,42=$ & 0,72 & 3,16 & 0,57 & ns \\
\hline
\end{tabular}

Los valores son promedio \pm desviación estándar.

más sedentaria que los niños normales, los métodos utilizados para estimar la condición física deben ser proporcionados a su situación, pues si bien es cierto que la determinacion directa del consumo máximo de oxígeno es el método más preciso, podría ser preferible en estos casos usar métodos indirectos, como el nomograma de Astrand validado para niños ${ }^{11}$ y el PWC-170 ${ }^{15}$, pues ambos son suficientemente sensibles a las modificaciones producidas por el entrenamiento aeróbico y cualquier sujeto, por sedentario que sea, es capaz de realizarlo en buena forma, evi. tando someter a niños poco habituados a esfuerzos agotadores, que podrían alejarlos aún más de la práctica de la actividad física. A pesar de todas las precauciones, hubo una deserción importante, hecho normal en este tipo de programas, principalmente cuando son de larga dura. ción. En los casos que describimos no se registró disminución de peso, sino aumento, el que es atribuible a crecimiento, por cuanto el porcen. taje de grasa magra disminuyó en forma significativa, como también de la suma del prosor de los pliegues cutáneos considerados. El entrena. miento físico determina aumento de la masa magra del cuerpo, asociado a disminución de la masa grasa, sin que se produzca obligadamente disminución del peso del individuo ${ }^{16}$. El aumento significativo de la capacidad de trabajo PWC-1 70 y el $\mathrm{VO}_{2}$ máx estimado demuestran que el entrenamiento logró su objetivo, al producir aumento de la capacidad fisica de los escolares participantes y, lo que es más importante, este fue acompañado de cambio de hábitos.

Los valores de colesterol total después de la aplicación del programa descendieron en forma significativa, confirmando la experiencia de Epstein $^{17}$. Sin embargo, a diferencia de sus resultados, no aumentó la fracción C-HDL, sino por el contrario, descendió. Es posible que el colesterol total tuviese una disminución importante, lo que traería como consecuencia concentracio- 
nes menores de las fracciones correspondientes, aunque también otros han encontrado descen. sos de las C-HDL ${ }^{18}$.

Los favorables resultados obtenidos en nuestro programa, posiblemente, obedecen a que está basado no sólo en recomendaciones dietarias y ejercicio, sino en lograr una modificación de la conducta. La mejoría obtenida en la incidencia de los factores de riesgo cardiovascular debería ser mantenida si se desea que tenga efectos a largo plazo. El aprendizaje de hábitos alimentarios correctos por el grupo familiar requiere la cooperación activa de los padres. Para ello, el contacto inicial debe ser mantenido, estimulando al nifro y enfatizando los beneficios de un programa de esta naturaleza.

\section{Resumen}

Se evaluaron la incidencia de factores de riesgo cardiovascular y el efecto de un programa de entrenamiento aeróbico de dos años de duración, en 30 niños obesos de ambos sexos y 6 a 12 años de edad. Sólo siete niños completaron el seguimiento. El entrenamiento consistió en tres sesiones semanales de ejercicios aeróbicos de $60 \mathrm{mi}-$ nutos de duración, buscando mantener 60 a $70 \%$ de la frecuencia cardíaca máxima calculada. Además se proporcionaron a los pacientes y sus padres información dietética $y$ recomendaciones tendientes a modificar los hábitos de alimentâción. Al término del estutio se registraron aumen. to de la capacidad de trabajo estimada para frecuencia cardíaca de $170 \cdot \min$ de 495 a 615 $\mathrm{kg} / \mathrm{min}$; disrnirnución del porcentaje de masa grasa corporal de 30,5 a $27,4 \%$; reducción del colesterol sérico total de 181 a $148 \mathrm{mg} / \mathrm{dl}$ y C-LDL de 106 a $84 \mathrm{mg} / \mathrm{dl}$, cambios todos significativos (p $<0,05)$.

(Palabras claves: Obesidad, lipoproteínas, colesterol, HDL, LDL, ejercicio.)

\section{Agradecimientos}

A las señoritas Olga Quintana G. y María Antonieta Rojas, por su contribución en el trabajo.

\section{Referencias}

1. Kagan A: Artherosclerosis of the aorta and coro. nary arteries in five towns. Bull World Health Organ $1976 ; 53: 485-645$.
2. Anónimo: Proyecto de Colaboración de la OMS Estudio sobre los precursores de la ateroesclerosis. Crónjca de la OMS 1980; 34: 206-208.

3. Hubert $H$, Feinleib $M$, McNamara $P$, Castelli $w$. Obesity as an independent risk factor for cardiovascular disease: A 26-year follow up of participants in the Framingham Heart Study: Circulation $1983 ; 67: 968-977$.

4. Milos $C$, Cosonueva $V$, Campos $R$, et al.: Factores de riesgo cardiovascular en una población de escolares chilenos. I parte, Lípidos séricos en 552 niños y adolescentes de 6 a 15 años. Rev Chil Pediatr 1990;61:67-74.

5. Chiong $M-T$, Milos $C$, Casamueve $V$, Cid $X$ : Perfil lipidico y capacidad de trabajo físico en escolases entienados y sedentarios obesos. Archivos Sociedad Chilena de Medicina del Deporte 1992; 37 15-20.

6. Notional Heart, Lung and Blood Institutes Task Force on Blood Pressure control in children. Report of Task Force on Blood Pressure Control. Pediatrics 1977; 59: 797-820.

7. Brook C: Determination of body composition of children from skinfold measurements. Arch Dis Child 1971;46: 182-186.

8. Sin $\boldsymbol{W}^{*}$ : Gross composition of the body. En: Ad. vances in biological and medical physics, New York, Academic Press 1957.

9. Avendafio A, Valenzuela $C$, Patri $A$ et al.: Antropometr ía de escolares chilenos del Area Norte de Santiago. Cuadernos Médico-Sociales (Santiago) $1975 ; 16: 5-12$

10. Passmore $R$, Durnin J: Human Energy Expenditure. Physial Rev 1955; 35: $801-820$.

11. Woynarawska $B$ : The validity of indirect estimations of maximal oxygen uptake in children 11-12 years of age. Eur J Appl Physiol 1980; 43; 19-23.

12. The Expert Panel: Report of the national cholesterol education program expert panel on detection, evaluation and treatment of high blood cholesterol in adults. Arch Int Med 1988; 148: 36-39.

13. De la Barra F: Aspectos psicosociales de la obesidad en el niño. Rev Chil Pediatr 1989; (Suppl) 60: $25-28$.

14. Commitree of Nutrition Am Acod Pediot: Prudent life style for children: Dietary fatand cholesterol. Pediattics 1986; $28: 521-524$.

15. Mocellin $R$, Limdemann $H$, Ruterfranz J, Sbrezny $X$ : Determination of PWC-170 and maximal oxygen uptake in children by different methods, Acta Paediatr Scand 1971; (Supp)). 217; 13-23.

16. Wilmore $J$ : Body composition in sport and exercise. Med Sci Sport Exerc 1983; 13: 21-23.

17. Epstein L. Kuller L, Wing L, Valoskj A, McCurley $J$ : The effect of weight control on lipid changes in obese children. Am J Djs Child 1989; 143: $450-457$.

18. Laskarzewsky P, Morrison J, Mellies $M$ : Relationship of measurements of body mass to plasma lipoproteins in schood children and adutts. Am J Epidemiol 1980; 111 ; 395-406. 